\title{
Clinical Significance of Molecular Biomarkers in Glioblastoma
}

\author{
C. Ang, M.-C. Guiot, A.V. Ramanakumar, D. Roberge, P. Kavan
}

\begin{abstract}
Aim: To review the impact of molecular biomarkers on response to therapy and survival in patients with primary glioblastoma (GBM). Materials \& Methods: Tissue specimens were analyzed for p53 mutations, EGFR amplification, loss of PTEN and p16, and $\mathrm{O}^{6}$-methylguanine DNA methyltransferase (MGMT) promoter methylation. Demographic and clinical data were gathered from medical records. Results: Clinical and pathological data of 125 patients were collected and analysed. MGMT promoter methylation was associated with improved median overall survival (OS) (61 vs. 42 weeks, p = 0.01) and was an important prognosticator independent of age at diagnosis, extent of resection and post-operative ECOG performance status (HR 2.04, 95\% CI 1.11-3.75). Among patients with MGMT promoter methylation, survival was significantly improved with chemoradiotherapy (CRT) over radiotherapy (RT) alone (71 vs. 14 weeks, p < 0.01). Furthermore, amongst those treated with temozolomide (TMZ) based CRT, the presence of EGFR amplification, maintenance of PTEN and wild-type p53 and p16 were each associated with trends towards improved survival. Conclusion: MGMT promoter methylation is a strong, independent prognostic factor for OS in GBM. EGFR amplification, maintenance of PTEN, wild-type p53 and p16 all appear to be associated with improved survival in patients treated with CRT. However, the prognostic value of these biomarkers could not be ascertained and larger prospective studies are warranted.
\end{abstract}

RÉSUMÉ: Signification clinique des biomarqueurs moléculaires dans le glioblastome. Objectif : Le but de l'étude était de réviser l'impact des biomarqueurs moléculaires sur la réponse au traitement et la survie chez les patients atteints de glioblastome primaire (GBP). Méthode : Des échantillons de tissus ont été analysés pour déterminer la présence de mutations du gène p53, d'amplification du gène de EGFR, de perte de PTEN et de p16 et de méthylation du promoteur de MGMT. Les données démographiques et cliniques ont été tirées des dossiers cliniques. Résultats : Les données cliniques et anatomopathologiques de 125 patients ont été recueillies et analysées. La méthylation du promoteur de MGMT était associée à une survie globale (SG) médiane améliorée, soit de 61 semaines par rapport à 42 semaines, $(\mathrm{p}=0,01)$ et était un facteur de pronostic important, indépendant de l'âge au moment du diagnostic, de l'étendue de la résection et de l'indice de performance postopératoire ECOG (RR 2,04; IC à 95\% 1,11 à 3,75). Parmi les patients qui avaient une méthylation du promoteur de MGMT, la survie était améliorée significativement avec la chimioradiothérapie (CRT) par rapport à la radiothérapie seule, soit 71 semaines par rapport à 14 semaines $(\mathrm{p}<0,01)$. De plus, parmi ceux qui avaient été traités par la CRT à base de témozolomide, la présence d'amplification de EGFR, la conservation de PTEN et de p53 et p16 de type sauvage étaient chacune associées à une tendance à une survie améliorée. Conclusion : La méthylation du promoteur de MGMT est un facteur de pronostic indépendant important pour la SG dans le GBM. L'amplification de EGFR, la conservation de PTEN, la présence de p53 et p16 de type sauvage semblent toutes associées à une meilleure survie chez les patients traités par la CRT. Cependant, la valeur de ces biomarqueurs n’a pu être déterminée et devra faire l'objet d'études prospectives plus considérables.

Can. J. Neurol. Sci. 2010; 37: 625-630

Glioblastoma (GBM) is the most common and aggressive intraparenchymal primary brain tumor in adults. Contemporary multimodality therapy for newly diagnosed patients, consisting of maximal surgical resection, concomitant radiation plus temozolomide (TMZ) followed by adjuvant $\mathrm{TMZ}^{1}$ has extended the median survival to 15 months, though only $10-26.5 \%$ of patients survive longer than two years. ${ }^{2}$ Treatment is challenging because of the notoriously unpredictable chemosensitivity of GBM which is likely linked to the genetic aberrations underlying its pathogenesis. Molecular biomarkers arising from these aberrations continue to be identified, and have been shown to have predictive and prognostic potential in GBM. In a post facto analysis of the landmark EORTC/NCIC study of adjuvant chemoradiation (CRT) with TMZ, it was suggested that methylation of the $\mathrm{O}^{6}$-methylguanine DNA methyltransferase
(MGMT) promoter is associated with sensitivity to TMZ and portends a favorable prognosis., ${ }^{2,3}$ Although the predictive and prognostic value of MGMT methylation status awaits further prospective validation, the EORTC/NCIC study established

\footnotetext{
From the Department of Oncology (CA, PK), McGill University and the Segal Cancer Centre - Sir Mortimer B. Davis Jewish General Hospital; Department of Pathology (MCG), McGill University Health Centre, Montreal Neurological Hospital; Division of Cancer Epidemiology (AVR), Department of Oncology, McGill University; Department of Radiation Oncology (DR), McGill University Health Centre, Montreal General Hospital, Montreal, Quebec, Canada.

Received December 22, 2009. Final Revisions Submitted March 3, 2010. Correspondence to: Petr Kavan, 3755 Rue Cote Ste Catherine, Pavillon E, Rm E-715 Jewish General Hospital, Montreal, Quebec, H3T 1E2, Canada.
} 
adjuvant CRT with TMZ followed by six months of maintenance TMZ as a new standard of care for the management of GBM and stimulated further interest in investigating other biomarkers.

Amplification of the epidermal growth factor receptor (EGFR) gene was one of the first genetic alterations identified in the pathogenesis of gliomas ${ }^{4}$ and is the target of the small molecule tyrosine kinase inhibitors (TKI) gefitinib and erlotinib. Investigations into the prognostic value of EGFR expression levels have produced mixed results, ${ }^{5-7}$ and the effect may be agedependent. ${ }^{8,9}$ Phosphatase tensin homolog on chromosome 10 (PTEN) is a tumor suppressor gene closely linked to the EGFR signalling pathway. ${ }^{10}$ Loss of PTEN, which occurs in $40-70 \%$ of GBM, ${ }^{11}$ results in uncontrolled cell proliferation and immortality ${ }^{10,12}$ independent of EGFR signalling and has been associated with poorer survival outcomes. ${ }^{11}$ The p53 tumor suppressor gene protects genetic integrity; mutations of $\mathrm{p} 53$ have been implicated in the development of many cancers. ${ }^{13}$ The prognostic role of p53 remains undefined in GBM, ${ }^{7,14}$ and its impact on response to therapy and survival has been shown to vary with age ${ }^{9}$ and may depend on which of its downstream effectors are affected. ${ }^{15,16}$ Finally, p16 is a key inhibitor in the cell cycle checkpoint regulating the transition from the G1 to $\mathrm{S}$ phase of DNA synthesis. ${ }^{17}$ A homozygous deletion of p16 is believed to represent a late step in the progression of astrocytic tumors into GBM. ${ }^{15}$ However, its clinical significance in GBM has yet to be established..$^{8,14}$

In view of these equivocal findings with respect to the predictive and prognostic role of various molecular biomarkers in the management of GBM, we decided to examine our own patient population with the hope of shedding further light on this issue. In this retrospective review, we describe the clinical and molecular profiles of patients diagnosed with primary GBM who were treated and followed at our institution, and evaluate their relationship with response to therapy and survival outcomes.

\section{Materials \& Methods}

Adult GBM patients operated on between January 1998 and January 2008 were identified in the pathology records of the Montreal Neurological Hospital. Only patients on whom molecular data were available were included in our review. Patients who were originally diagnosed with lower grade gliomas which transformed into secondary GBM were excluded. Patient demographics, treatment regimens and clinical outcomes were abstracted from hospital charts.

Tissue specimens were processed and analyzed for specific molecular markers. Genomic DNA was isolated from $15 \mu \mathrm{m}$ thick paraffin sections using the QIAamp DNA mini kit (Qiagen Sciences, MD, USA). Bisulfite treatment of $1 \mu \mathrm{g}$ of DNA was performed using the EZ DNA Methylation Gold Kit (Zymo Research, Orange, CA, USA). ${ }^{18}$ Methylation of the MGMT promoter gene was assessed by methylation specific polymerase chain reaction (PCR). This was performed using the specific primers to distinguish methylated and unmethylated DNA. ${ }^{19}$ The primer sequences used were as follows: UM forward 5'TTTGTGTTTTGATGTTTGTAGGTTTTTGT 3', UM reverse 5'AACTCСАСАСТСТTCCAAАAАCAAAACA 3', M forward 5'TTTCGACGTTCGTA GGTTTTCGC 3' and $M$ reverse GCACTCTTCCGAAAACGAAACG 3'. Methylated and unmethylated PCR products were visualized on a Criterion precast gel 10\% TBE (Bio-Rad Laboratories, Inc., CA, USA). Control DNAs were treated concomitantly and consisted of genomic DNA isolated from the HeLa cell line for the methylated form and from placental DNA for the unmethylated form.

Amplification of EGFR, loss of p16 and PTEN were assessed by dual-color interphasic fluorescence in situ hybridization. This was performed on $5 \mu \mathrm{m}$ thick formalin fixed paraffin embedded sections using commercially fluoresecent probe sets from Vysis (Abbott Molecular, IL, USA) and following the manufacturer protocol: LSI 1p36/LSI1q25, LSI19q13/19p13, LSI EGFR/CEP7, LSI p16/CEP9 and LSI PTEN (10q23)/CEP10. p53 mutations were assessed by the fluorescent single-strand conformation polymorphism (SSCP) assay using an ABI 310 genetic analyzer.

Kaplan and Meier survival analysis was used to compute the survival time. Overall survival (OS) was calculated from the index date of biopsy or resection to the date of death or last follow-up. Time to progression (TTP) was determined from the date of pathological diagnosis to the date of first radiographic or clinical evidence of disease recurrence, progression or death. Differences in OS and TTP due to biomarkers as well as the known clinical parameters of age ( $>50$ years, $<50$ years), extent of surgery (resection vs. biopsy), post-operative Eastern Cooperative Oncology Group (ECOG) performance status (0-2 vs. 3+) and adjuvant CRT with TMZ vs. Adjuvant radiation (RT) alone were compared using the log-rank test. The impact of biomarkers on survival outcomes was calculated using the Cox proportional hazards model after adjusting for the aforementioned clinical parameters. Statistical analyses were performed using SPSS (SPSS Inc., Chicago, IL, USA) and STATA 10.1 (Stata Corp LP, TX, USA) statistical software.

\section{Results}

\section{Patient Demographics}

Molecular data were available on a total of 137 initially identified patients. Of these, 12 were excluded from our analysis. Nine patients had developed a secondary GBM from a previous lower grade glioma and three patients lacked basic demographic and clinical data including date of birth, date and extent of surgery or last follow-up. The clinical characteristics of the remaining 125 patients are presented in Table 1.

\section{Management}

Data on patient management are also summarized in Table 1. Of the 122 patients for whom operative reports were retrieved, $101(83 \%)$ underwent surgical resection of their tumors. Seventy-eight (62\%) patients received radiation (40-60 Gy) concurrent with TMZ followed by maintenance TMZ for a median of four months (1-12 months). Within the CRT group, three patients actually underwent sequential, but not concurrent, radiation followed by TMZ. One patient within the CRT group received CPT-11 on the RTOG 0420 protocol. Twenty-five (20\%) patients were treated with RT alone at doses of 20-60Gy. Of the remaining 22 patients, two received RT concurrent with gefitinib on the Radiation Therapy Oncology Group (RTOG) 0211 protocol, one patient underwent RT followed by carmustine, and two patients received TMZ monotherapy. Six 
Table 1: Patient demographic \& clinical characteristics

\begin{tabular}{l|l}
\hline Characteristic $(\mathbf{N}=\mathbf{1 2 5})$ & $\mathbf{N}$ \\
\hline Male:Female & $74: 51$ \\
\hline $\begin{array}{l}\text { Age } \leq 50:>50 \\
\text { Median age: } 60 \text { (range 15-86) }\end{array}$ & $30: 95$ \\
\hline ECOG 0-2:3+ & $56: 8(61$ missing) \\
\hline Resection:biopsy & $101: 21$ (3 missing) \\
\hline CRT with TMZ:RT only & $78: 25$ \\
\hline
\end{tabular}

$\mathrm{CRT}=$ chemoradiotherapy $\mathrm{RT}=$ radiotherapy;

$\mathrm{TMZ}=$ temozolomide; $\mathrm{ECOG}=$ Eastern Cooperative Oncology Group

patients were treated with comfort measures postoperatively, two patients died before adjuvant therapy began, three refused adjuvant therapy, and one had an intracranial hemorrhage postoperatively thereby precluding further treatment. Treatment information was missing for the remaining five patients.

Seventy-four (59\%) patients had documented relapses. Of the patients on whom information on second line therapy was available, 15 were salvaged with surgery alone and two were treated with stereotactic radiosurgery at a dose of $15 \mathrm{~Gy}$. Others were treated with surgery followed by various regimens of salvage chemotherapy as outlined in Table 2 .

\section{Molecular Biomarkers}

Of the 105 patients whose tumors were evaluable for MGMT promoter methylation status $57(54 \%)$ had methylated promoters. Epidermal growth factor receptor expression was assessed in 112 patients and was amplified in 46 (41\%) cases. Phosphatase tensin homolog on chromosome 10 was lost in 52 $(65 \%)$ of 80 tested cases. Out of 77 samples tested for the p16 regulatory protein, 33 (43\%) contained a homozygous deletion. p53 was mutated in nine (29\%) of 31 tumor samples amenable to testing. These results are summarized in Table 3 .

\section{Clinical Characteristics \& Survival Outcomes}

The median survival time for the entire study cohort was 53 weeks (95\% confidence interval [CI] 47.7-58.3 weeks). Median time to progression was 25 weeks (95\% CI 20.8-29.2 weeks). Median survival post relapse was 27 weeks (95\% CI 19.7-34.2). Median duration of follow-up in living patients was 73 weeks (range 1-199 weeks).

Surgical resection and a postoperative ECOG performance status of $<2$ were associated with trends towards better median OS (53 vs. 38 weeks, $\mathrm{p}=0.39$ and 51 vs. 38 weeks, $\mathrm{p}=0.87$, respectively). Age at diagnosis ( $>50$ years vs. $\leq 50$ years-of-age) and sex were not important clinical prognosticators. On univariate analysis, treatment TMZ based CRT resulted in a trend towards improved median OS compared to those treated with RT alone (60 vs. 38 weeks, $\mathrm{p}=0.18)$. These findings are
Table 2: Second-line chemotherapy regimens

\begin{tabular}{l|l}
\hline Chemotherapy & N \\
\hline PCV (procarbazine, lomustine, vincristine) & 3 \\
\hline lomustine & 1 \\
\hline alternative dose TMZ monotherapy & 17 \\
\hline TMZ + procarbazine & 18 \\
\hline lapatinib & 1 \\
\hline TMZ + bevacizumab & 1 \\
\hline carmustine (Gliadel $^{\top M}$ ) wafer & 2 \\
\hline TMZ + procarbazine + bevacizumab & 1 \\
\hline
\end{tabular}

$\mathrm{TMZ}=$ temozolomide

summarized in Table 4. On multivariate analysis adjusting for age, sex and postoperative ECOG performance status, tumor resection instead of biopsy (HR 2.43, 95\% CI 1.11-5.31), and CRT as opposed to RT alone (HR 4.42, 95\% CI 2.14-9.13), emerged as significantly prognostic of improved survival. There was insufficient clinical data to subdivide our patient population into the recursive partitioning analysis subgroups previously described by Curran et al. ${ }^{20}$

\section{Impact of Molecular Biomarkers on Survival}

Methylation of the MGMT promoter was associated with a markedly improved OS time (61 vs. 42 weeks, $p=0.01)$ as well as TTP (30 vs. 24 weeks, $p=0.02$ ). In the subgroup of patients

Table 3: Biomarker distribution

\begin{tabular}{l|l}
\hline Biomarker & $\mathbf{N}$ \\
\hline EGFR amplified:nonamplified & $46: 66$ \\
\hline MGMT methylated:unmethylated & $57: 48$ \\
\hline PTEN hemideletion:no loss & $52: 28$ \\
\hline p16 homozygous deletion:no loss & $33: 44$ \\
\hline p53 mutated:nonmutated & $9: 22$ \\
\hline
\end{tabular}

EGFR = epidermal growth factor receptor; $\mathrm{MGMT}=\mathrm{O}^{6}$-methylguanine DNA methyltransferase; PTEN = phosphatase tensin homolog on chromosome 10 
who received CRT only, those with a methylated MGMT promoter benefited significantly (71 vs. 42 weeks, $\mathrm{p}<0.01$ ). Amongst those with MGMT promoter methylation, treatment with CRT resulted in a highly significant increase in OS compared to RT alone (71 vs. 14 weeks, $\mathrm{p}<0.01$; Figure).

Among patients who received CRT, EGFR amplification was associated with a longer median OS (70 vs. 54 weeks, $\mathrm{p}=0.07$ ). Similar but weaker trends were also observed among patients in the CRT subgroup who had maintenance of PTEN (74 vs. 59 weeks, $\mathrm{p}=0.12$ ), wild-type p53 (67 vs. 53 weeks, $\mathrm{p}=0.35$ ) or wild-type p16 (67 vs. 54 weeks, p = 0.24). However, when the entire study population was examined, EGFR amplification status, loss of PTEN, and mutations of p16 and p53 did not appreciably affect OS or TTP. These findings are summarized in Table 5.

Cox regression analysis confirmed the strong prognostic potential of MGMT methylation status. Patients with an unmethylated MGMT promoter experienced a higher risk of mortality compared to those with a methylated promoter (HR $1.72,95 \%$ CI 1.13-2.62). This effect was maintained after adjusting for the potential confounding effects of age at diagnosis, extent of resection and postoperative performance status (HR 2.04, 95\% CI 1.11-3.75). The prognostic value of EGFR, PTEN, p53 and p16 were not established in the current analysis.

\section{Discussion}

In this retrospective study, the importance of MGMT promoter methylation status was confirmed. MGMT promoter methylation was associated with a significantly longer median OS, even after adjusting for established clinical prognosticators such as age, extent of resection and performance status. ${ }^{20}$ The current study also suggests a predictive effect of MGMT promoter methylation status on sensitivity to CRT compared to

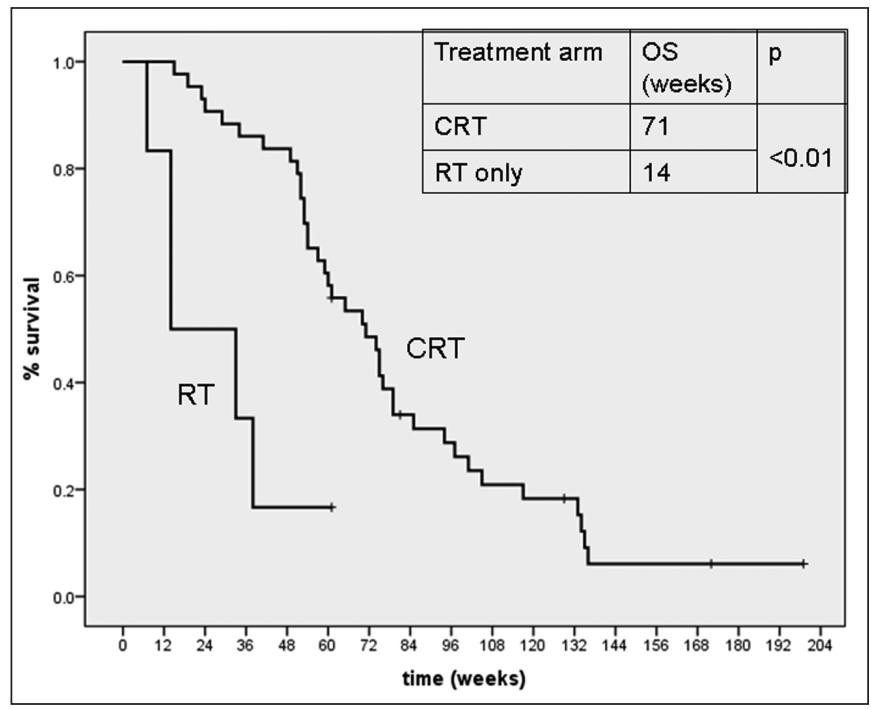

Figure: $O S$ by treatment arm in patients with MGMT promoter methylation. $C R T=$ chemoradiotherapy; $R T=$ radiotherapy; $O S=$ overall survival; $M G M T=O^{6}$-methylguanine DNA methyltransferase
Table 4: Impact of clinical and treatment characteristics on survival

\begin{tabular}{l|l|l|l}
\hline Characteristic & N & Median OS (weeks) & p \\
\hline $\begin{array}{l}\text { Age } \\
\leq 50 \text { years }\end{array}$ & 30 & 53 & \\
$>50$ years & 95 & 52 & 0.41 \\
\hline $\begin{array}{l}\text { Sex } \\
\text { Female }\end{array}$ & 51 & 53 & \\
Male & 74 & 53 & 0.44 \\
\hline $\begin{array}{l}\text { Extent of Surgery } \\
\text { Resection }\end{array}$ & 101 & 53 & \\
Biopsy & 21 & 38 & 0.39 \\
\hline $\begin{array}{l}\text { Postoperative ECOG } \\
\text { 0-2 }\end{array}$ & 56 & 51 & \\
3+ & 8 & 38 & 0.87 \\
\hline $\begin{array}{l}\text { Adjuvant Treatment } \\
\text { CRT with TMZ }\end{array}$ & 78 & 60 & \\
RT only & 25 & 38 & 0.18 \\
\hline
\end{tabular}

$\mathrm{CRT}=$ chemoradiotherapy $\mathrm{RT}=$ radiotherapy $\mathrm{TMZ}=$ temozolomide

RT alone. However, it is becoming evident that other factors may also influence survival outcomes on TMZ as even patients with an unmethylated MGMT promoter also appear to have a survival benefit with CRT. ${ }^{3}$ For example, variations in expression levels of mutated p53 and MGMT have been associated with differences in progression free survival time on TMZ. ${ }^{21}$

With respect to the impact of other molecular biomarkers, among patients who received CRT, those with EGFR amplification, maintained PTEN, wild-type p16 or p53 tended to experience longer survival times. In a phase II study combining erlotinib with TMZ based CRT, OS appeared to be improved compared to historical controls. ${ }^{22}$ Furthermore, patients with an intact PTEN as well as MGMT promoter methylation experienced a significant survival advantage, ${ }^{22}$ although the mechanism underlying this observation has yet to be elucidated. In view of the respective roles of PTEN and MGMT in regulating cell proliferation and DNA repair, it is conceivable that, in the rapidly dividing and error-prone environment of tumor cells, PTEN maintenance and MGMT promoter silencing would produce a favourable phenotype.

The prognostic impact of EGFR has been less clear; examination of EGFR expression levels have produced contradictory results. ${ }^{5-7}$ EGFR and PTEN activity are closely intertwined through the PI3K/Akt signalling cascade and the mutation of either compound will result in the constitutive activation of this pathway. ${ }^{12}$ To our knowledge, no studies, including the current one, have specifically examined the effect of PTEN or EGFR on response to CRT with TMZ. Although the results in the current study are intriguing, they must be interpreted with caution especially since they were derived from a small and heterogeneous study population.

It was surprising to observe that none of the clinical characteristics, including established prognosticators such as 
Table 5: Impact of biomarkers on survival outcomes

\begin{tabular}{|c|c|c|c|c|}
\hline Biomarker (N) & $\begin{array}{l}\text { Median OS } \\
\text { (weeks) }\end{array}$ & $\mathbf{p}$ & $\begin{array}{l}\text { Median TTP } \\
\text { (weeks) }\end{array}$ & $\mathbf{p}$ \\
\hline $\begin{array}{l}\text { MGMT (105) } \\
\text { Methylated } \\
\text { Unmethylated }\end{array}$ & \begin{tabular}{|l|}
61 \\
42 \\
\end{tabular} & 0.01 & $\begin{array}{l}30 \\
24\end{array}$ & 0.02 \\
\hline $\begin{array}{l}\text { EGFR (112) } \\
\text { Amplified } \\
\text { Nonamplified } \\
\end{array}$ & $\begin{array}{l}59 \\
52\end{array}$ & 0.63 & $\begin{array}{l}25 \\
27 \\
\end{array}$ & 0.45 \\
\hline $\begin{array}{l}\text { PTEN (80) } \\
\text { Hemideletion } \\
\text { No loss }\end{array}$ & $\begin{array}{l}52 \\
53 \\
\end{array}$ & 0.38 & $\begin{array}{l}29 \\
23 \\
\end{array}$ & 0.72 \\
\hline $\begin{array}{l}\text { p16 (77) } \\
\text { Homozygous deletion } \\
\text { No loss }\end{array}$ & $\begin{array}{l}44 \\
54 \\
\end{array}$ & 0.11 & $\begin{array}{l}21 \\
29 \\
\end{array}$ & 0.09 \\
\hline $\begin{array}{l}\mathrm{p} 53(31) \\
\text { Mutated } \\
\text { Nonmutated }\end{array}$ & $\begin{array}{l}38 \\
34\end{array}$ & 0.46 & $\begin{array}{l}23 \\
25\end{array}$ & 0.30 \\
\hline
\end{tabular}

EGFR $=$ epidermal growth factor receptor; MGMT $=\mathrm{O}^{6}$-methylguanine DNA methyltransferase; PTEN = phosphatase tensin homolog on chromosome 10

extent of resection and performance status, were observed to have a significant impact on survival. In the case of extent of surgery, a possible explanation might be heterogeneity and the absence of standardized or quantitative definitions of what constitutes a gross total, subtotal or partial resection. Assignment of the type of resection was made based upon operative reports and not post-operative imaging. In the case of performance status, this was likely an issue of insufficient power given that only six patients had an ECOG $\geq 3$ compared to 51 patients with an ECOG of 0-2. The absence of a significant difference between patients treated with CRT versus RT only may also be explained by sample heterogeneity. Furthermore, it is important to note that patients who relapsed but who maintained an adequate performance status were eligible for second, and even third-line treatments with various combinations of surgery, local and systemic therapy. However, given the small size of our study population, it was not possible to examine how salvage therapy might have interacted with our biomarker analysis, or to ascertain the extent to which it influenced the observed outcomes.

A key concept in oncology is that of the pluripotent cancer stem cell. Tumorigenesis arises from a common progenitor cell through the accumulation and complex interplay of mutations in key signalling pathways regulating growth, differentiation and apoptosis. In many cancers, it has become apparent that the molecular byproducts of these mutations have relevance as potential targets for drug development or predictors of response to therapy. This has driven the shift in therapeutic strategies in oncology from "traditional" cytotoxic chemotherapy, which remains indispensable in cancer treatment, to molecular pathway-specific targeted agents. In GBM, there has been evidence to suggest that primary, secondary and perhaps recurrent disease are each characterized by distinct gene expression profiles which, in turn, appear to correlate with differential sensitivities to various chemotherapeutic and targeted agents. For example, primary GBM is typically associated with EGFR amplification and deletion of PTEN, while p53 inactivation is more commonly seen with secondary GBM. ${ }^{23-25}$ While retrospective studies examining the relationship between EGFR amplification status and response to erlotinib have produced contradictory results, ${ }^{22,26}$ expression of its mutant variant EGFRvIII, has been associated with sensitivity to gefitinib in the recurrent setting. ${ }^{12,27}$ The presence or absence of PTEN, in conjunction with EGFR or EGFRvIII expression, also appears to modulate sensitivity to TKI in recurrent GBM. ${ }^{12,28,29}$ Clearly, GBM is a disease with extremely heterogeneous clinical and molecular properties that have been shown to interact with each other. ${ }^{8}$ For these reasons, the search for the optimal approach to utilizing and integrating this information in a clinically meaningful way continues to be a challenging endeavour.

\section{CONCLUSION}

$\mathrm{O}^{6}$-methylguanine DNA methyltransferase promoter methylation status is a strong, independent prognostic factor for overall survival in GBM. EGFR amplification, maintenance of PTEN, and the presence of wild-type p53 and p16 each appear to be associated with improved survival in patients treated with CRT. However, with the exception of MGMT, the prognostic value of these biomarkers could not be ascertained in a statistically meaningful way and larger prospective studies may be warranted. In the absence of more effective therapies directed towards specific molecular variations, the utility of routine genetic profiling in GBM will remain limited.

\section{ACKNOWLEDGEMENTS}

The authors thank Dr. Eduardo Franco from the Division of Cancer Epidemiology for his assistance with our statistical analysis.

\section{DisClosures}

Dr. Kavan received an unrestricted educational grant from Schering-Plough, Canada. Dr. Roberge received research funding and has also served as a consultant for Schering-Plough, Canada.

\section{REFERENCES}

1. Stupp R, Mason WP, van den Bent MJ, Weller M, Fisher B, Taphoorn MJ, et al. Radiotherapy plus concomitant and adjuvant temozolomide for glioblastoma. N Engl J Med. 2005; 352(10):987-96.

2. Wen PY, Kesari S. Malignant gliomas in adults. N Engl J Med. 2008;359(5):492-507.

3. Hegi ME, Diserens AC, Gorlia T, Hamou MF, de Tribolet N, Weller $\mathrm{M}$, et al. MGMT gene silencing and benefit from temozolomide in glioblastoma. N Engl J Med. 2005;352(10):997-1003.

4. Liberman TA, Nussbaum HR, Razon N, Kris R, Lax I, Soreq H, et al. Amplification, enhanced expression and possible rearrangement of EGF receptor gene in primary human brain tumours of glial origin. Nature. 1985;313(5998):144-7.

5. Quaranta M, Divella R, Daniele A, Di Tardo S, Venneri MT, Lolli I, et al. Epidermal growth factor serum levels and prognostic value in malignant gliomas. Tumori. 2007;93(3):275-80. 
6. Wagner M, Menei P, Guilhot J, Levillain P, Michalak S, Bataille B, et al. Prognostic molecular markers with no impact on decision making: the paradox of gliomas based on a prospective study. $\mathrm{Br}$ J Cancer. 2008;98(11):1830-8.

7. Houillier C, Lejeune J, Benouaich-Amiel A, Laigle-Donadey F, Criniere E, Mokhtari K, et al. Prognostic impact of molecular markers in a series of 220 primary glioblastoma. Cancer. 2006; 106(10):2218-23.

8. Korshunov A, Sycheva R, Golanov A. The prognostic relevance of molecular alterations in glioblastomas for patients age $<50$ years. Cancer. 2005;104(4):825-32.

9. Batchelor TT, Betensky Ra, Esposito JM, Pham LD, Dorfman MV, Piscatelli N, et al. Age-dependent prognostic effects of genetic alterations in glioblastoma. Clin Cancer Res. 2004;10(1 Pt 1): 228-33.

10. Bianco R, Shin I, Ritter CA, Yakes FM, Basso A, Rosen N, et al. Loss of PTEN/MMAC1/TEP in EGF receptor-expressing tumor cells counteracts the antitumor action of EGFR tyrosine kinase inhibitors. Oncogene. 2003;22(18):2812-22.

11. Koul D. PTEN signalling pathways in glioblastoma. Cancer Biol Ther. 2008;7(9):1321-5.

12. Mellinghoff IK, Wang MY, Vivanco I, Haas-Kogan DA, Zhu S, Dia $\mathrm{EQ}$, et al. Molecular determinants of the response of glioblastomas to EGFR kinase inhibitors. N Engl J Med. 2005; 353(19):2012-24.

13. Foulkes WD. Inherited susceptibility to common cancers. N Engl J Med. 2008;359(20):2143-53.

14. Newcomb EW, Cohen H, Lee SR, Bhalla SK, Bloom J, Hayes RL, et al. Survival of patients with glioblastoma multiforme is not influenced by altered expression of p16, p53, EGFR, MDM2 or Bcl-2 genes. Brain Pathol. 1998;8(4):655-67.

15. Nozaki M, Tada M, Kobayashi H, Zhang CL, Sawamura Y, Abe H, et al. Roles of the functional loss of p53 and other genes in astrocytoma tumorigenesis and progression. Neuro Oncol. 1999; $1(2): 124-37$.

16. Yount GL, Haas-Kogan DA, Vidair CA, Haas M, Dewey WC, Israel MA. Cell cycle synchrony unmasks the influence of $\mathrm{p} 53$ function on radiosensitivity of human glioblastoma cells. Cancer Res. 1996;56(3):500-6.

17. Nakamura K, Watanabe T, Klangby U, Asker C, Wiman K, Yonekawa Y, et al. p14Arf deletion and methylation in genetic pathways to glioblastomas. Brain Pathol. 2001;11(2):159-68.
18. Cankovic M, Mikkelsen T, Rosenblum RL, Zarbo RJ. A simplified laboratory validated assay for MGMT promoter hypermethylation analysis of glioma specimens from formalin-fixed paraffin-embedded tissue. Lab Invest. 2007;87(4):392-7.

19. Esteller M, Hamilton SR, Burger PC, Baylin SB, Herman JG. Inactivation of the DNA repair gene $\mathrm{O}^{6}$-methylguanine-DNA methyltransferase by promoter hypermethylation is a common event in primary human neoplasia. Cancer Res. 1999;59(4): 793-7.

20. Curran WJ Jr, Scott CB, Horton J, Nelson JS, Weinstein AS, Fischbach AJ, et al. Recursive partitioning analysis of prognostic factors in three Radiation Therapy Oncology Group malignant glioma trials. J Natl Cancer Inst. 1993;85(9):704-10.

21. Li S, Jiang T, Li G, Wang Z. Impact of p53 status to response of temozolomide in low MGMT expression glioblastoma: preliminary results. Neurol Res. 2008;30(6):567-70.

22. Prados MD, Chang SM, Butowski N, DeBoer R, Parvataneni R, Carliner $\mathrm{H}$, et al. Phase II study of erlotinib plus temozolomide during and after radiation therapy in patients with newly diagnosed glioblastoma multiforme or gliosarcoma. J Clin Oncol. 2009;27(4):579-84.

23. Ohgaki H, Kleihues P. Genetic pathways to primary and secondary glioblastoma. Am J Pathol. 2007;170(5):1445-53.

24. Mischel PS, Nelson SF, Cloughesy TF. Molecular analysis of glioblastoma: pathway profiling and its implications for patient therapy. Cancer Biol Ther. 2003;2(3):242-8.

25. Kleihues P, Ohgaki H. Primary and secondary glioblastoma: from concept to clinical diagnosis. Neuro-Oncol. 1999;1(1):44-51.

26. De Groot JF, Gilbert MR, Aldape K, Hess KR, Hanna TA, Ictech S, et al. Phase II study of carboplatin and erlotinib (Tarceva, OSI774) in patients with recurrent glioblastoma. J Neurooncol. 2008;90(1):89-97.

27. Rich JN, Reardon DA, Peery T, Dowell JM, Quinn JA, Penne KL, et al. Phase II trial of gefitinib in recurrent glioblastoma. J Clin Oncol. 2004;22(1):133-42.

28. Haas-Kogan DA, Prados MD, Tihan T, Eberhard DA, Jelluma N, Arvold ND, et al. Epidermal growth factor receptor, protein kinase B/Akt, and glioma response to erlotinib. J Natl Cancer Inst. 2005;97(12):880-7.

29. Huang F, Kavan P, Guiot M-C, Markovic Y, Roberge D. When temozolomide alone fails: adding procarbazine in salvage therapy of glioma. Can J Neurol Sci. 2008;35(2):192-7. 